\title{
ANALYTIC HIERARCHY PROCESS BEST APPROACH IN SEQUENCING OF ORDINARY DISTILLATION COLUMNS
}

*Note: Do not include the author(s) names and information as this document will be blind reviewed and they will be entered during proposal submission.

\begin{abstract}
Analytic hierarchy process (AHP) ratings mode were applied combined with heuristics rules for choosing the best sequence of ordinary distillation columns in a five component mixture. Categories for each heuristic according to its compliance in each sequence, were defined and pairwise compared. At the same time, the heuristics rules were equally weighted to cancel conflict among them. The AHP ratings mode approach proved to be effective in selecting the best alternative, compared against the results obtained with more complex and rigorous methods.
\end{abstract}

Keywords: AHP ratings mode, distillation column sequences.

\section{Introduction}

In chemical process design, the need to separate multicomponent mixtures into essentially pure products arise, and sequences of ordinary distillation columns are preferred to meet this need. However, the number of different sequences that can do the separation task grows in a number-of-component factorial way. Even when there are heuristics rules known, several studies had shown that they usually are in conflict with each other, particularly when try to define which of them is more important (Seider, 1999). This study solves the conflict of heuristics rules using AHP (Saaty, 2008).

\section{Literature Review}

The sequencing ordinary distillation columns problem could be found in several chemical process design textbooks (Seider, 1999), and had been linked to rather complex AHP applications (AHP + genetic algorithms or hybrid models) in Li et. al. (2009) and Cziner et. al. (2006). So, the simple approach used in this work has not been implemented yet.

\section{Hypotheses/Objectives}

This study proves that the combination of AHP ratings mode with heuristics rules can be a suitable and not contradictory approach in the ordinary distillation columns sequencing.

\section{Research Design/Methodology}

Even when there are several more comprehensive methods for ordinary distillation sequencing, the most common method used involves heuristics rules. The most common heuristics rules are the following four: 1) Do the most difficult separation last; 2) Remove pure products one by one as distillates (direct sequences); 3) Remove first component 
with largest percentage of the feed; 4) Favor near equimolar amounts of distillates and bottoms in each column, (Seider, 1999). So, the goal of the model is to select the distillation sequence that best fit to these four heuristics rules, which in turn become the criteria, and finally, the alternatives in the model correspond to the different distillation column sequences. Example 5.2 (Seider, 1999) was used as case study, which involves the separation of a five component mixture with fourteen different sequences to choice. Even when applying the heuristics rules to the different sequences results in some numerical data, it was preferred to use ratings mode of AHP with personal interpretation of what those numbers mean. On the other hand, it was assigned the same weight to the four heuristics, to cancel the alleged conflict between them.

\section{Data/Model Analysis}
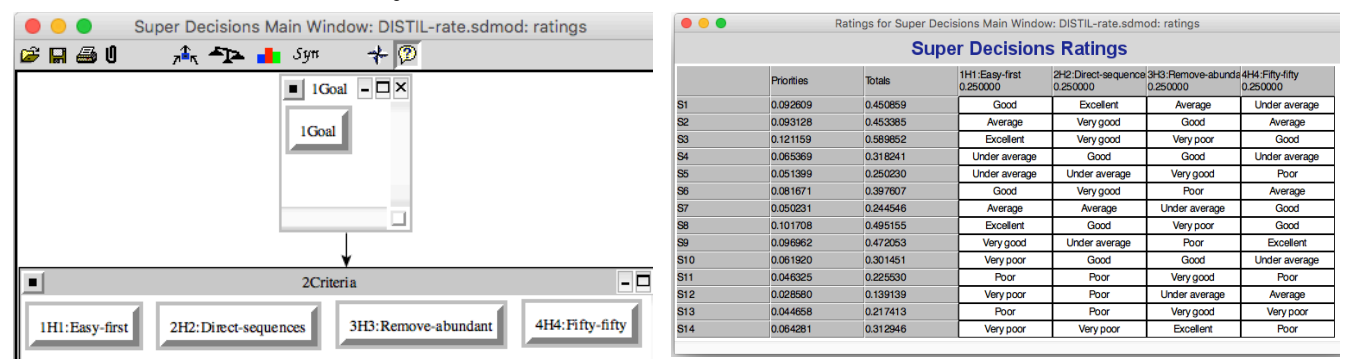

The left figure shows the AHP ratings model used in Super Decisions ${ }^{\circledR}$ software. The right figure shows the ratings matrix of the heuristics rules. CI for the rules ratings were: $\mathrm{H} 1=0.0930, \mathrm{H} 2=0.0993, \mathrm{H} 3=0.0314$, and $\mathrm{H} 4=0.0324$. The preferred sequence is $\mathrm{S} 3$, which coincides with the chosen as optimal with more rigorous methods (Seider, 1999).

\section{Limitations}

The visualization of all different sequences, which facilitates the application of the AHP ratings mode, becomes cumbersome as the number of pure products grows.

\section{Conclusions}

The results allow to illustrate that, even though in some cases the heuristics rules seem contradictory, if they are used combined with AHP ratings mode, can be a suitable initial guidance on the problems of ordinary distillation sequencing, so it is advisable to teach the heuristics rules method complemented with the application of the AHP ratings mode.

\section{Key References}

Cziner, K., Hassim, M., \& Hurme, M. (2006). Multicriteria Design of Separation Sequences by Including HSE Criteria and Uncertainty. $16^{\text {th }}$ ESCAPE.

Li, C., Wozny, G., \& Suzuki, K. (2009). Design and synthesis of separation process based on a hybrid method. Asia-Pacific Journal of Chemical Engineering, 4, 905-915.

Saaty, T.L. (2008). Decision making with the analytic hierarchy process. Int. J. Services Sciences, 1, 83-98.

Seider, W.D., Seader, J.D., \& Lewin, D.R. (1999). Process Design Principles. New York, USA: John Wiley \& Sons, Inc. 